\title{
New results for perturbed second-order impulsive differential equation on the half-line
}

\author{
Yulin Zhao, Xuebin Wang ${ }^{*}$ and Xingguo Liu
}

"Correspondence: xuebingw@126.com

School of Science, Hunan University of Technology, Zhuzhou, Hunan 412007, People's Republic of China

\begin{abstract}
By using a variational method and some critical points theorems, we establish some results on the multiplicity of solutions for second-order impulsive differential equation depending on two real parameters on the half-line. In addition, two examples to illustrate our results are given.
\end{abstract}

Keywords: impulsive differential equation; variational methods; critical points; half-line

\section{Introduction}

In this paper we consider the following boundary value problem with impulsive effects:

$$
\left\{\begin{array}{l}
-u^{\prime \prime}(t)+m^{2} u(t)=\lambda f(t, u(t)), \quad t \in[0,+\infty), t \neq t_{j} \\
\triangle\left(u^{\prime}\left(t_{j}\right)\right)=\mu I_{j}\left(u\left(t_{j}\right)\right), \quad j=1,2, \ldots, p \\
u^{\prime}\left(0^{+}\right)=g(u(0)), \quad u^{\prime}(+\infty)=0
\end{array}\right.
$$

where $m$ is a non-zero constant; $\lambda$ and $\mu$ are referred to as two control parameters, $I_{j} \in C(\mathbf{R}, \mathbf{R})$ for $1 \leq j \leq p, 0=t_{0}<t_{1}<t_{2}<\cdots<t_{p}<+\infty, \Delta\left(u^{\prime}\left(t_{j}\right)\right)=u^{\prime}\left(t_{j}^{+}\right)-u^{\prime}\left(t_{j}^{-}\right)=$ $\lim _{t \rightarrow t_{j}^{+}} u^{\prime}(t)-\lim _{t \rightarrow t_{j}^{-}} u^{\prime}(t) ; f:[0,+\infty) \times \mathbf{R} \rightarrow \mathbf{R}$ is an $L^{2}$-Carathéodory function, and $g: \mathbf{R} \rightarrow \mathbf{R}$ be a Lipschitz continuous function with the Lipschitz constant $L>0$; i.e.

$$
\left|g\left(s_{1}\right)-g\left(s_{2}\right)\right| \leq L\left|s_{1}-s_{2}\right|
$$

for all $s_{1}, s_{2} \in \mathbf{R}$, satisfying $g(0)=0$.

Boundary value problems on the half-line, arising naturally in the study of radially symmetric solutions of nonlinear elliptic equations and various physical phenomena [1], have been studied extensively and a variety of new results can be found in the papers [2-6] and the references cited therein. Criteria for the existence of solutions or multiplicities of positive solutions are established for the boundary value problem on the half-line. The main tools used in the literature for such a problem are the coincidence degree theory of Mawhin, fixed point arguments together with the lower and upper solutions method. For example the readers are referred to [1-8] and the references therein.

Recently, many researchers pay more attention to the impulsive boundary value problems, such as Dirichlet boundary value problem, periodic boundary value problem, two

\section{黛 Springer}

(c) 2014 Zhao et al.; licensee Springer. This is an Open Access article distributed under the terms of the Creative Commons Attribution License (http://creativecommons.org/licenses/by/4.0), which permits unrestricted use, distribution, and reproduction in any medium, provided the original work is properly credited. 
point boundary value problem and so on (see for example, $[9,10]$ and references therein). The existence or multiplicity of solutions for impulsive boundary value problems (IBVP) on the half-line has been studied by many authors [11-14]. Kaufmann et al. [13] investigated the following impulsive boundary value problem:

$$
\left\{\begin{array}{l}
-\left(q u^{\prime}\right)^{\prime}(t)=f(t, u(t)), \quad t \in[0,+\infty), t \neq t_{j}, \\
\triangle\left(u^{\prime}\left(t_{j}\right)\right)=I_{j}\left(u\left(t_{j}\right)\right), \quad j=1,2, \ldots, p, \\
\alpha u^{\prime}(0)-\beta \lim _{t \rightarrow 0^{+}} q(t) u^{\prime}(t)=0, \\
\gamma \lim _{t \rightarrow \infty} u(t)+\delta \lim _{t \rightarrow \infty} q(t) u^{\prime}(t)=0,
\end{array}\right.
$$

where $q \in C[0, \infty) \cap C^{1}[0, \infty)$ and $q(t)>0$ for all $t>0$; and $f:[0,+\infty) \times \mathbf{R} \rightarrow \mathbf{R}$ is continuous. By using the fixed point theorem, the existence of at least one solution for IBVP (1.3) is obtained.

In [14], Li and Nieto considered the existence of multiple positive solutions of the following IBVP on the half-line:

$$
\left\{\begin{array}{l}
u^{\prime \prime}(t)+q(t) f(t, u(t))=0, \quad t \in[0,+\infty), t \neq t_{j}, \\
\triangle\left(u\left(t_{j}\right)=I_{j}\left(u\left(t_{j}\right)\right), \quad j=1,2, \ldots, p,\right. \\
\alpha u(0)=\sum_{i=1}^{m-2} \alpha_{i} u\left(\xi_{i}\right), \quad \lim _{t \rightarrow \infty} u^{\prime}(t)=0
\end{array}\right.
$$

where $q \in C([0, \infty),[0, \infty))$ and $f:[0,+\infty) \times[0,+\infty) \rightarrow[0,+\infty)$ is continuous. By using a fixed point theorem due to Avery and Peterson, the existence of at least three positive solutions is obtained.

On the other hand, critical point theory and variational methods are proved to be a powerful tool in studying the existence of solutions for the impulsive differential equations [15-28]. For some recent works on the theory of critical point theory and variational methods we refer the readers to [29].

In the case $m=\mu=1$, Chen and Sun [17] studied and presented some results on the existence and multiplicity of solutions for IBVP (1.1) by using a variational method and a three critical points theorem due to Bonanno and Marano (see Theorem 2.1 of [30]). The result is as follows.

Theorem 1.1 ([17, Theorem 3.1]) Suppose that the following conditions hold.

(H1) $g(u), I_{j}(u)$ are nondecreasing, and $g(u) u \geq 0, I_{j}(u) u \geq 0$ for any $u \in \mathbf{R}$.

(H2) There exist positive constants $a, l$ with $l<2, b \in L^{1}[0,+\infty)$ and $c \in L^{2}[0,+\infty)$ such that

$$
F(t, u) \leq b(t)\left(a+|u|^{l}\right), \quad f(t, u) \leq c(t)|u|^{l-1}, \quad \text { for a.e. } t \in[0,+\infty) \text { and all } u \in \mathbf{R},
$$

where $F(t, u)=\int_{0}^{u} f(t, s) d s$.

(H3) There exist two constants $d, k>0$ such that

$$
\frac{d^{2}}{M^{2}}<k^{2}+2 \sum_{j=1}^{p} \int_{0}^{k e^{-t_{j}}} I_{j}(s) d s+2 \int_{0}^{k} g(s) d s .
$$

(H4) $\frac{M^{2} \int_{0}^{+\infty} \max _{|\xi| \leq d} F(t, \xi) d t}{d^{2}}<\frac{\int_{0}^{+\infty} F\left(t, k e^{-t}\right) d t}{k^{2}+2 \sum_{j=1}^{p} \int_{0}^{k e^{-t j}} I_{j}(s) d s+2 \int_{0}^{k} g(s) d s}$. 
Then, for each $\lambda \in\left[\frac{\frac{k^{2}}{2}+\sum_{j=1}^{p} \int_{0}^{k e^{-t}} I_{j}(s) d s+\int_{0}^{k} g(s) d s}{\int_{0}^{+\infty} F\left(t, k e^{-t}\right) d t}, \frac{d^{2}}{2 M^{2} \int_{0}^{+\infty} \max _{|\xi| \leq d} F(t, \xi) d t}\right], I B V P(1.1)$ has at least three classical solutions.

Soon after, in the case $m=\mu=1$, by using the variant fountain theorems (see Theorem 2.2 of [31]), Dai and Zhang [25] obtained some existence theorems of solutions for IBVP (1.1) when the function $g$ and the impulsive functions $I_{j}(j=1,2, \ldots, p)$ satisfies the following superlinear growth conditions:

$\left(\mathrm{H} 1^{\prime}\right) I_{j}(u)(j=1,2, \ldots, p), g(u)$ satisfy $\int_{0}^{u} I_{j}(s) d s \geq 0, \int_{0}^{u} g(s) d s \geq 0$ for any $u \in \mathbf{R}$; and there exist positive constants $\gamma_{j}>\alpha-1, \theta>\alpha-1(1<\alpha<2)$ and $q_{j}, q(j=1,2, \ldots, p)$ such that $I_{j}(u) \leq q_{j}|u|^{\gamma_{j}}, g(u) \leq q|u|^{\theta}, \forall u \in \mathbf{R}$.

However, there is no work for IBVP (1.1) when the parameter $\mu \not \equiv 1$ and $f$ is an $L^{2}$ Carathéodory function. As a result, the goal of this paper is to fill the gap in this area. Our aim is to establish a precise open interval $\Lambda \subseteq(0,+\infty)$, for each $\lambda \in \Lambda$, there exists a $\delta>0$ such that for each $\mu \in[0, \delta)$, IBVP (1.1) admits at least three classical solutions.

The remainder of the paper is organized as follows. In Section 2, we present some preliminaries. In Section 3, we will state and prove the main results of the paper, and also two examples are presented to illustrate our main results.

\section{Preliminaries}

In this section, we first introduce some notations and some necessary definitions.

Set

$W:=\left\{u:[0,+\infty) \rightarrow \mathbf{R}\right.$ is absolutely continuous, $\left.u^{\prime} \in L^{2}[0,+\infty)\right\}$

Denote the Sobolev space $X$ by

$$
X:=\left\{u \in W: \int_{0}^{+\infty}\left(\left|u^{\prime}(t)\right|^{2}+m^{2}|u(t)|^{2}\right) d t<\infty\right\}
$$

with the inner product

$$
(u, v)=\int_{0}^{+\infty}\left(u^{\prime}(t) v^{\prime}(t)+m^{2} u(t) v(t)\right) d t
$$

which induces the norm

$$
\|u\|_{X}:=\left(\int_{0}^{+\infty}\left(\left|u^{\prime}(t)\right|^{2}+m^{2}|u(t)|^{2}\right) d t\right)^{1 / 2}
$$

Obviously, $X$ is a reflexive Banach space. We define the norm in $L^{2}([0,+\infty))$ as $\|u\|_{2}=$ $\left(\int_{0}^{\infty}|u(t)|^{2} d t\right)^{1 / 2}$, and let $\mathcal{C}:=\left\{u \in C([0,+\infty)): \sup _{t \in[0,+\infty)}|u(t)|<+\infty\right\}$, with the norm $\|u\|_{\infty}=\sup _{t \in[0,+\infty)}|u(t)|$. Then $\mathcal{C}$ is a Banach space. In addition, $X$ is continuously embedded into $\mathcal{C}$, and therefore, there exist two constants $M_{1}, M_{2}$ such that

$$
\|u\|_{\infty} \leq M_{1}\|u\|_{X}, \quad \text { for any } u \in X,
$$


and

$$
\|u\|_{2} \leq M_{2}\|u\|_{X}, \quad \text { for any } u \in X
$$

Suppose that $u \in C[0,+\infty)$. By a classical solution of IBVP (1.1), we mean a function

$$
\begin{aligned}
u \in & \left\{z \in C([0,+\infty)):\left.z\right|_{\left[t_{j}, t_{j+1}\right]} \in C^{2}\left(\left[t_{j}, t_{j+1}\right]\right), j=0,1,2, \ldots, p-1 ;\right. \\
& \left.\left.z\right|_{\left[t_{p},+\infty\right)} \in C^{2}\left(\left[t_{p},+\infty\right)\right)\right\}
\end{aligned}
$$

that satisfies the equation in IBVP (1.1) a.e. on $[0,+\infty)$, the limits $u^{\prime}\left(t_{j}^{+}\right), u^{\prime}\left(t_{j}^{-}\right), j=1,2, \ldots, p$ exist and the impulsive conditions in IBVP $(1.1)$ hold; $u^{\prime}\left(0^{+}\right), u^{\prime}(+\infty)$ exist, and the boundary conditions in IBVP (1.1) also are met.

For each $u \in X$, put

$$
\begin{aligned}
& \Phi(u)=\frac{1}{2}\|u\|_{X}^{2}+G(u(0)), \\
& \Psi(u)=\int_{0}^{+\infty} F(t, u(t)) d t-\frac{\mu}{\lambda} \sum_{j=1}^{p} \int_{0}^{u\left(t_{j}\right)} I_{j}(s) d s,
\end{aligned}
$$

where $G(u(0))=\int_{0}^{u(0)} g(s) d s, F(t, u)=\int_{0}^{u} f(t, s) d s$.

It is clear that $\Psi$ is differentiable at any $u \in X$ and

$$
\Psi^{\prime}(u)(v)=\int_{0}^{+\infty} f(t, u(t)) v(t) d t-\frac{\mu}{\lambda} \sum_{j=1}^{p} I_{j}\left(u\left(t_{j}\right)\right) v\left(t_{j}\right)
$$

for any $v \in X$.

Recall that a function $f:[0,+\infty) \times \mathbf{R} \rightarrow \mathbf{R}$ is said to be an $L^{2}$-Carathéodory function, if

(S1) $t \rightarrow f(t, u)$ is measurable for every $u \in \mathbf{R}$;

(S2) $u \rightarrow f(t, u)$ is continuous for almost every $t \in[0,+\infty)$;

(S3) for every $\rho>0$ there exists a function $l_{\rho} \in L^{2}([0,+\infty))$ such that

$$
\sup _{|u| \leq \rho}|f(t, u)| \leq l_{\rho}(t), \quad \text { for almost every } t \in[0,+\infty) \text {. }
$$

If we assume that the function $f$ satisfies the further condition

$\left(\mathrm{S}^{\prime}\right)$ there exists a function $l \in L^{2}([0,+\infty))$ such that

$$
\sup _{u \in \mathbf{R}}|f(t, u)| \leq l(t), \quad \text { for almost every } t \in[0,+\infty)
$$

then one has the following result.

Lemma 2.1 Suppose that condition (S3') holds. Then $\Psi^{\prime}: X \rightarrow X^{*}$ is a compact operator. In particular, $\Psi: X \rightarrow \mathbf{R}$ is a weakly sequentially continuous functional.

Proof Let $\Omega$ be a bounded set in $X$ and let $\left\{a_{n}\right\}$ be a sequence in $\overline{\Psi^{\prime}(\Omega)}$. Then there is a sequence $\left\{u_{n}\right\}$ in $\Omega$ such that $b_{n}=\Psi^{\prime}\left(u_{n}\right)$ and $\left\|a_{n}-b_{n}\right\|_{X^{*}}<\frac{1}{n}$ for all $n \in \mathbf{N}$. Due to $X$ being reflexive, there exists a subsequence $u_{n_{k}}$ converging weakly to $u \in X$. We can prove that 
$\left\{u_{n}\right\}$ has a subsequence which converges almost everywhere in $[0,+\infty)$ to the function $u$. In fact, given a number $r>0$, let $\Omega_{r}:=\Omega([0, r))$. Then we easily infer that $\left\{u_{n}\right\}$ is bounded in $\Omega_{r}$. Pick $r=1$, the Rellich-Kondrachov theorem [32, Theorem IX.16] yields a subsequence $\left\{u_{n}^{(1)}\right\}$ of $\left\{u_{n}\right\}$ such that $\lim _{n \rightarrow \infty} u_{n}^{(1)}(t)=u(t)$ at most all points $t \in[0,1)$. Applying this argument again, with 1 replaced by 2 , we also obtain a sequence $\left\{u_{n}^{(2)}\right\}$ of $\left\{u_{n}\right\}$ such that $\lim _{n \rightarrow \infty} u_{n}^{(2)}(t)=u(t)$ at almost all points $t \in[0,2)$. Thus, the sequence $\left\{u_{n}^{(n)}\right\}$ clearly complies with the conclusion. Without loss of generality we write $\left\{u_{n}^{(n)}\right\}$ as $\left\{u_{n_{k}}\right\}$. Therefore, $\left\{f\left(t, u_{n_{k}}\right)\right\}$ converges to $\{f(t, u(t))\}$ a.e. on $[0, \infty)$. From (2.1), (2.2), and (2.5), we have

$$
\begin{aligned}
& \left|\Psi^{\prime}\left(u_{n_{k}}\right)(v)-\Psi^{\prime}(u)(v)\right| \\
& \leq \int_{0}^{\infty}\left|f\left(t, u_{n_{k}}(t)\right)-f(t, u(t))\right| \cdot|v(t)| d t+\frac{\mu}{\lambda} \sum_{j=1}^{p}\left|I_{j}\left(u_{n_{k}}\left(t_{j}\right)\right)-I_{j}\left(u\left(t_{j}\right)\right)\right| \cdot\left|v\left(t_{j}\right)\right| \\
& \leq\left(\int_{0}^{\infty}\left|f\left(t, u_{n_{k}}(t)\right)-f(t, u(t))\right|^{2} d t\right)^{1 / 2} \cdot\left(\int_{0}^{\infty}|v(t)|^{2} d t\right)^{1 / 2} \\
& \quad+\frac{\mu}{\lambda} \sum_{j=1}^{p}\left|I_{j}\left(u_{n_{k}}\left(t_{j}\right)\right)-I_{j}\left(u\left(t_{j}\right)\right)\right| \cdot\|v\|_{\infty} \\
& \leq M_{2}\left(\int_{0}^{\infty}\left|f\left(t, u_{n_{k}}(t)\right)-f(t, u(t))\right|^{2} d t\right)^{1 / 2}+\frac{\mu}{\lambda} M_{1} \sum_{j=1}^{p}\left|I_{j}\left(u_{n_{k}}\left(t_{j}\right)\right)-I_{j}\left(u\left(t_{j}\right)\right)\right|
\end{aligned}
$$

for all $v \in X$ with $\|v\|_{X} \leq 1$. Hence, from (S3'), the Lebesgue dominated convergence theorem and continuity of $I_{j}$ show that the sequence $\left\{\Psi^{\prime}\left(u_{n_{k}}\right)\right\}$ converges to $\Psi^{\prime}(u)$ in $X^{*}$. Therefore, taking into account that

$$
\left\|a_{n_{k}}-\Psi^{\prime}(u)\right\|_{X^{*}} \leq\left\|a_{n_{k}}-b_{n_{k}}\right\|_{X^{*}}+\left\|b_{n_{k}}-\Psi^{\prime}(u)\right\|_{X^{*}},
$$

the sequence $\left\{a_{n_{k}}\right\}$ converges in $\overline{\Psi^{\prime}(\Omega)}$ and the compactness is proved.

Finally, it follows from Corollary 41.9 of [33, p.236] that $\Psi$ is a weakly sequentially continuous functional. This completes the proof.

By standard arguments, we find that $\Phi$ is a Gâteaux differentiable and sequentially weakly lower semicontinuous functional whose Gateaux derivative at the point $u \in X$ is the functional $\Phi^{\prime}(u) \in X^{*}$, given by

$$
\Phi^{\prime}(u)(v)=\int_{0}^{+\infty}\left(u^{\prime}(t) v^{\prime}(t)+m^{2} u(t) v(t)\right) d t+g(u(0)) v(0)
$$

for any $v \in X$.

Definition 2.2 A function $u \in X$ is said to be a weak solution IBVP (1.1) if $u$ satisfies

$$
\begin{aligned}
& \int_{0}^{+\infty} u^{\prime}(t) v^{\prime}(t) d t+m^{2} \int_{0}^{+\infty} u(t) v(t) d t-\lambda \int_{0}^{+\infty} f(t, u(t)) v(t) d t \\
& +\mu \sum_{j=1}^{p} I_{j}\left(u\left(t_{j}\right)\right) v\left(t_{j}\right)+g(u(0)) v(0)=0,
\end{aligned}
$$

for any $v \in X$. 
It is easy to verify that $u \in X$ is a weak solution to IBVP (1.1) if and only if $u$ is a classical solution of IBVP (1.1).

Arguing in a standard way, it is easy to prove that the critical points of the functional $E_{\lambda}:=\Phi(u)-\lambda \Psi(u)$ are the weak solution of IBVP (1.1) and so they are classical solutions.

The main tools to prove our results in Section 3 are the following critical points theorems.

Theorem 2.3 ([18]) Let $X$ be a reflexive real Banach space; $\Phi: X \rightarrow \mathbf{R}$ be a sequentially weakly lower semicontinuous, coercive and continuously Gâteaux differentiable functional whose Gâteaux derivative admits a continuous inverse on $X^{*}, \Psi: X \rightarrow \mathbf{R}$ be a sequentially weakly upper semicontinuous, continuously Gâteaux differentiable functional whose Gâteaux derivative is compact, such that $\Phi(0)=\Psi(0)=0$. Assume that there exist $r>0$ and $\bar{v} \in X$, with $r<\Phi(\bar{v})$ such that

(i) $\sup _{\Phi(u) \leq r} \Psi(u)<r \Psi(\bar{v}) / \Phi(\bar{v})$,

(ii) for each $\lambda$ in

$$
\Lambda_{r}:=\left(\frac{\Phi(\bar{v})}{\Psi(\bar{v})}, \frac{r}{\sup _{\Phi(u) \leq r} \Psi(u)}\right),
$$

the functional $\Phi-\lambda \Psi$ is coercive. Then, for each $\lambda \in \Lambda_{r}$ the functional $\Phi-\lambda \Psi$ has at least three distinct critical points in $X$.

Theorem 2.4 ([34]) Let $X$ be a reflexive real Banach space; $\Phi: X \rightarrow \mathbf{R}$ be a convex, coercive and continuously Gâteaux differentiable functionals whose derivative admits a continuous inverse on $X^{*}, \Psi: X \rightarrow \mathbf{R}$ be a continuously Gâteaux differentiable functionals whose derivative is compact, such that

(1) $\inf _{X} \Phi=\Phi(0)=\Psi(0)=0$;

(2) for each $\lambda>0$ and for every $u_{1}, u_{2}$ which are local minimum for the functional $\Phi-\lambda \Psi$ and such that $\Psi\left(u_{1}\right) \geq 0$ and $\Psi\left(u_{2}\right) \geq 0$, one has

$$
\inf _{s \in[0,1]} \Psi\left(s u_{1}+(1-s) u_{2}\right) \geq 0
$$

Assume that there are two positive constants $r_{1}, r_{2}$, and $\bar{v} \in X$, with $2 r_{1}<\Phi(\bar{v})<\frac{r_{2}}{2}$, such that

(b1) $\frac{\sup _{u \in \Phi^{-1}\left(-\infty, r_{1}\right)} \Psi(u)}{r_{1}}<\frac{2 \Psi(\bar{v})}{3 \Phi(\bar{v})}$;

(b2) $\frac{\sup _{u \in \Phi^{-1}\left(-\infty, r_{2}\right)} \Psi(u)}{r_{2}}<\frac{\Psi(\bar{v})}{3 \Phi(\bar{v})}$.

Then, for each $\lambda \in\left(\frac{3 \Phi(\bar{v})}{2 \Psi(\bar{v})}, \min \left\{\frac{r_{1}}{\sup _{u \in \Phi^{-1}\left(-\infty, r_{1}\right)} \Psi(u)}, \frac{r_{2}}{2 \sup _{u \in \Phi^{-1}\left(-\infty, r_{2}\right)} \Psi(u)}\right\}\right)$, the functional $\Phi-\lambda \Psi$ has at least three distinct critical points which lie in $\Phi^{-1}\left(-\infty, r_{2}\right)$.

\section{Main results}

Lemma 3.1 Suppose that

(C0) there exist constants $\alpha_{j}, \beta_{j}>0$, and $\sigma_{j} \in[0,1)(j=1,2, \ldots, p)$ such that

$$
\left|I_{j}(u)\right| \leq \alpha_{j}+\beta_{j}\left|u\left(t_{j}\right)\right|^{\sigma_{j}}, \quad \text { for any } u \in \mathbf{R}, j=1,2, \ldots, p \text {. }
$$


Then, for each $u \in X$, we have

$$
\left|\sum_{j=1}^{p} \int_{0}^{u\left(t_{j}\right)} I_{j}(s) d s\right| \leq \sum_{j=1}^{p}\left(\alpha_{j}\|u\|_{\infty}+\frac{\beta_{j}}{\sigma_{j}+1}\|u\|_{\infty}^{\sigma_{j}+1}\right) .
$$

Proof By the condition (C0), we have

$$
\left|\int_{0}^{u\left(t_{j}\right)} I_{j}(s) d s\right| \leq \alpha_{j}\left|u\left(t_{j}\right)\right|+\frac{\beta_{j}}{\sigma_{j}+1}\left|u\left(t_{j}\right)\right|^{\sigma_{j}+1} .
$$

Thus, (3.1) is proved.

Now we can state and prove our main results.

Theorem 3.2 Assume that (C0) and $M_{1}^{2} L<1$ hold. Let $f:[0,+\infty) \times \mathbf{R} \rightarrow \mathbf{R}$ be an $L^{2}$ Carathéodory function such that (S3') satisfies. Furthermore, suppose that there exist two positive constants $a$ and $b$ such that

(C1) $a^{2}<\frac{M_{1}^{2} b^{2}\left(m^{2}-2 M_{1}^{2} L+1\right)}{2}$;

(C2) $\frac{\int_{0}^{+\infty} F\left(t, b e^{-t}\right) d t}{b^{2}\left(m^{2}+2 M_{1}^{2} L+1\right)}>\frac{M_{1}^{2} \cdot\left(\int_{0}^{+\infty} \sup _{|\xi| \leq a}|f(t, \xi)|^{2} d t\right)^{1 / 2}}{2 a\left(1-M_{1}^{2} L\right)}$, and $\int_{0}^{+\infty} F\left(t, b e^{-t}\right) d t>0$.

Then, for each $\lambda$ in

$$
\Lambda_{1}:=\left(\frac{b^{2}\left(m^{2}+2 M_{1}^{2} L+1\right)}{4 \int_{0}^{+\infty} F\left(t, b e^{-t}\right) d t}, \frac{\left(1-M_{1}^{2} L\right) a}{2 M_{1}^{2} \cdot\left(\int_{0}^{+\infty} \sup _{|\xi| \leq a}|f(t, \xi)|^{2} d t\right)^{1 / 2}}\right)
$$

there exists

$$
\begin{aligned}
\delta_{1}:= & \min \left\{\frac{\left(1-M_{1}^{2} L\right) a-2 M_{1}^{2} \lambda \cdot\left(\int_{0}^{+\infty} \sup _{|\xi| \leq a}|f(t, \xi)|^{2} d t\right)^{1 / 2}}{2 M_{1}^{2}\left(\sum_{j=1}^{p}\left(\alpha_{j} a+\frac{\beta_{j}}{\sigma_{j}+1} a^{\sigma_{j}+1}\right)\right)},\right. \\
& \left.\frac{4 \lambda \int_{0}^{+\infty} F\left(t, b e^{-t}\right) d t-b^{2}\left(m^{2}+2 M_{1}^{2} L+1\right)}{4 \sum_{j=1}^{p}\left(\alpha_{j} b+\frac{\beta_{j}}{\sigma_{j}+1} b^{\sigma_{j}+1}\right)}\right\}
\end{aligned}
$$

such that for each $\mu \in\left[0, \delta_{1}\right), I B V P(1.1)$ has at least three distinct classical solutions.

Proof Obviously, under the condition $\left(\mathrm{S3}^{\prime}\right), \Psi: X \rightarrow \mathbf{R}$ is weakly sequentially lower semicontinuous and Gâteaux differentiable functional.

Note that as (1.2) holds for every $s_{1}, s_{2} \in \mathbf{R}$ and $g(0)=0$, one has

$$
|g(s)|=|g(s)-g(0)| \leq L|s|, \quad \text { for every } s \in \mathbf{R} \text {. }
$$

Furthermore, for any $u \in X$, one has

$$
\Phi(u)=\frac{1}{2}\|u\|_{X}^{2}+\int_{0}^{u(0)} g(s) d s \leq \frac{1}{2}\|u\|_{X}^{2}+\int_{0}^{u(0)} L|s| d s \leq \frac{1+M_{1}^{2} L}{2}\|u\|_{X}^{2}
$$

and

$$
\Phi(u) \geq \frac{1}{2}\|u\|_{X}^{2}-\int_{0}^{u(0)}|g(s)| d s \geq \frac{1}{2}\|u\|_{X}^{2}-\int_{0}^{u(0)} L|s| d s \geq \frac{1-M_{1}^{2} L}{2}\|u\|_{X}^{2} .
$$

So $\Phi$ is coercive. 
Next, we show that $\Phi^{\prime}: X \rightarrow X^{*}$ admits a Lipschitz continuous inverse. For any $u \in$ $X \backslash\{0\}$, it follows from (2.6) and (1.2) that

$$
\left\langle\Phi^{\prime}(u), u\right\rangle=\int_{0}^{+\infty}\left(\left|u^{\prime}(t)\right|^{2}+m^{2}|u(t)|^{2}\right) d t+g(u(0)) u(0) \geq\left(1-M_{1}^{2} L\right)\|u\|_{X}^{2}
$$

By the assumption $M_{1}^{2} L<1$, it turns out that

$$
\lim _{\|u\|_{X} \rightarrow+\infty} \frac{\left\langle\Phi^{\prime}(u), u\right\rangle}{\|u\|_{X}}=+\infty
$$

that is, $\Phi^{\prime}$ is coercive.

For any $u, v \in X$,

$$
\begin{aligned}
\left\langle\Phi^{\prime}(u)-\Phi^{\prime}(v), u-v\right\rangle & =\langle u-v, u-v\rangle+(g(u(0))-g(v(0)))(u(0)-v(0)) \\
& \geq\left(1-M_{1}^{2} L\right)\|u-v\|_{X}^{2},
\end{aligned}
$$

so $\Phi^{\prime}$ is a strongly monotone operator. By [35, Theorem 26.A], one finds that $\left(\Phi^{\prime}\right)^{-1}$ exists and is Lipschitz continuous on $X^{*}$. Hence the functionals $\Phi$ and $\Psi$ satisfy the regularity assumptions of Theorem 2.3.

Furthermore for any fixed $\lambda$, and $\mu$ as in (3.2), (3.3). Put $r=\frac{\left(1-M_{1}^{2} L\right) a^{2}}{2 M_{1}^{2}}$. Taking (2.1) into account, for every $u \in X$ such that $\Phi(u) \leq r$, we have $\sup _{t \in[0,+\infty)}|u(t)| \leq a$. Therefore, it follows from (2.4), (3.1), and the Hölder inequality that

$$
\begin{aligned}
\sup _{\Phi(u) \leq r} \Psi(u) & =\sup _{\Phi(u) \leq r}\left\{\int_{0}^{+\infty} F(t, u(t)) d t-\frac{\mu}{\lambda} \sum_{j=1}^{p} \int_{0}^{u\left(t_{j}\right)} I_{j}(s) d s\right\} \\
& \leq \sup _{\Phi(u) \leq r}\left\{\int_{0}^{+\infty} \sup _{|\xi| \leq a}|f(t, \xi)||u(t)| d t+\frac{\mu}{\lambda} \sum_{j=1}^{p}\left(\alpha_{j}\|u\|_{\infty}+\frac{\beta_{j}}{\sigma_{j}+1}\|u\|_{\infty}^{\sigma_{j}+1}\right)\right\} \\
& \leq \sup _{\Phi(u) \leq r}\left(\int_{0}^{+\infty} \sup _{|\xi| \leq a}|f(t, \xi)|^{2} d t\right)^{1 / 2} \cdot\|u\|_{2}+\frac{\mu}{\lambda} \sum_{j=1}^{p}\left(\alpha_{j} a+\frac{\beta_{j}}{\sigma_{j}+1} a^{\sigma_{j}+1}\right) \\
& \leq a \cdot\left(\int_{0}^{+\infty} \sup _{|\xi| \leq a}|f(t, \xi)|^{2} d t\right)^{1 / 2}+\frac{\mu}{\lambda} \sum_{j=1}^{p}\left(\alpha_{j} a+\frac{\beta_{j}}{\sigma_{j}+1} a^{\sigma_{j}+1}\right),
\end{aligned}
$$

which implies

$$
\sup _{\Phi(u) \leq r} \frac{\Psi(u)}{r} \leq \frac{2 M_{1}^{2}}{\left(1-M_{1}^{2} L\right) a}\left\{\cdot\left(\int_{0}^{+\infty} \sup _{|\xi| \leq a}|f(t, \xi)|^{2} d t\right)^{1 / 2}+\frac{\mu}{\lambda} \sum_{j=1}^{p}\left(\alpha_{j}+\frac{\beta_{j}}{\sigma_{j}+1} a^{\sigma_{j}}\right)\right\} .
$$

Since $\mu<\delta_{1}$, one has

$$
\sup _{\Phi(u) \leq r} \frac{\Psi(u)}{r}<\frac{1}{\lambda}
$$


Put $v_{0}(t)=b e^{-t}$. Obviously, $v_{0} \in X,\left\|v_{0}\right\|^{2}=\frac{\left(m^{2}+1\right) b^{2}}{2}$. By a similar reasoning to (3.4) and (3.5), we get

$$
\frac{m^{2}-2 M_{1}^{2} L+1}{4} b^{2} \leq \Phi\left(v_{0}\right) \leq \frac{2 M_{1}^{2} L+m^{2}+1}{4} b^{2} .
$$

From $(\mathrm{C} 1)$ we get $r<\Phi\left(v_{0}\right)$. It follows from (2.4) that

$$
\begin{aligned}
\Psi\left(v_{0}\right) & =\int_{0}^{+\infty} F\left(t, v_{0}(t)\right) d t-\frac{\mu}{\lambda} \sum_{j=1}^{p} \int_{0}^{v_{0}\left(t_{j}\right)} I_{j}(s) d s \\
& \geq \int_{0}^{+\infty} F\left(t, v_{0}(t)\right) d t-\frac{\mu}{\lambda} \sum_{j=1}^{p}\left(\alpha_{j}\left\|v_{0}\left(t_{j}\right)\right\|_{\infty}+\frac{\beta_{j}}{\sigma_{j}+1}\left\|v_{0}\left(t_{j}\right)\right\|_{\infty}^{\sigma_{j}+1}\right) \\
& \geq \int_{0}^{+\infty} F\left(t, b e^{-t}\right) d t-\frac{\mu}{\lambda} \sum_{j=1}^{p}\left(\alpha_{j} b+\frac{\beta_{j}}{\sigma_{j}+1} b^{\sigma_{j}+1}\right),
\end{aligned}
$$

then

$$
\frac{\Psi\left(v_{0}\right)}{\Phi\left(v_{0}\right)} \geq \frac{\int_{0}^{+\infty} F\left(t, b e^{-t}\right) d t-\frac{\mu}{\lambda} \sum_{j}^{p}\left(\alpha_{j} b+\frac{\beta_{j}}{\sigma_{j}+1} b^{\sigma_{j}+1}\right)}{\frac{\left(m^{2}+2 M_{1}^{2} L+1\right) b^{2}}{4}} .
$$

Since $\mu<\delta_{1}$, one has

$$
\frac{\Psi\left(v_{0}\right)}{\Phi\left(v_{0}\right)}>\frac{1}{\lambda}
$$

Combining with (3.6) and (3.7), condition (i) of Theorem 2.3 is fulfilled.

Next we will prove the coercivity of the functional $E_{\lambda}$.

Taking into account $\left(\mathrm{S}^{\prime}\right)$ and the Hölder inequality, one has

$$
\begin{aligned}
\int_{0}^{+\infty} F(t, u(t)) d t & \leq \int_{0}^{+\infty}\left(\int_{0}^{u} \sup _{\xi \in \mathbf{R}}|f(t, \xi)| d \xi\right) d t \\
& \leq \int_{0}^{+\infty}\left(\int_{0}^{u} l(t) d \xi\right) d t \\
& \leq \int_{0}^{+\infty}|l(t) \| u(t)| d t \\
& \leq\left(\int_{0}^{+\infty} l^{2}(t) d t\right)^{1 / 2} \cdot\left(\int_{0}^{+\infty}|u(t)|^{2} d t\right)^{1 / 2} \\
& =\|l\|_{2} \cdot\|u\|_{2}<+\infty,
\end{aligned}
$$

combining with (2.2) and Lemma 3.1, it follows that

$$
\begin{aligned}
E_{\lambda}(u) & =\Phi(u)-\lambda \Psi(u) \\
& =\frac{1}{2}\|u\|_{X}^{2}+G(u(0))-\lambda \int_{0}^{+\infty} F(t, u(t)) d t+\mu \sum_{j=1}^{p} \int_{0}^{u\left(t_{j}\right)} I_{j}(s) d s
\end{aligned}
$$




$$
\begin{aligned}
\geq & \frac{1-M_{1}^{2} L}{2}\|u\|_{X}^{2}-\lambda \int_{0}^{+\infty}\left(\int_{0}^{u} \sup _{\xi \in \mathbf{R}} f(t, \xi) \mid d \xi\right) d t \\
& -\mu \sum_{j=1}^{p}\left(\alpha_{j}\|u\|_{\infty}+\frac{\beta_{j}}{\sigma_{j}+1}\|u\|_{\infty}^{\sigma_{j}+1}\right) \\
\geq & \frac{1-M_{1}^{2} L}{2}\|u\|_{X}^{2}-\lambda\|l\|_{2} \cdot\|u\|_{2}-\mu \sum_{j=1}^{p}\left(\alpha_{j} M_{1}\|u\|_{X}+\frac{\beta_{j} M_{1}^{\sigma_{j}+1}}{\sigma_{j}+1}\|u\|_{X}^{\sigma_{j}+1}\right) \\
\geq & \frac{1-M_{1}^{2} L}{2}\|u\|_{X}^{2}-\left(\lambda M_{2}\|l\|_{2}+\mu M_{1} \sum_{j=1}^{p} \alpha_{j}\right)\|u\|_{X}-\mu \sum_{j=1}^{p} \frac{\beta_{j} M_{1}^{\sigma_{j}+1}}{\sigma_{j}+1}\|u\|_{X}^{\sigma_{j}+1} .
\end{aligned}
$$

Since $M_{1}^{2} L<1,1 \leq \sigma_{j}+1<2$, the above inequality implies that $\lim _{\|u\|_{X} \rightarrow+\infty} E_{\lambda}(u)=+\infty$, so $E_{\lambda}(u)$ is coercive. According to Theorem 2.3, it follows that, for each

$$
\lambda \in \Lambda_{1}
$$

the functional $E_{\lambda}(u)=\Phi(u)-\lambda \Psi(u)$ has at least three distinct critical points, i.e. IBVP (1.1) has at least three distinct weak solutions. This completes the proof.

Let

$$
I_{r}^{*}:=\sum_{j=1}^{p} \min _{|\xi| \leq r} \int_{0}^{\xi} I_{j}(s) d s, \quad \text { for any } r>0
$$

and

$$
\theta=\frac{2 M_{1}^{2}}{1-M_{1}^{2} L} .
$$

Theorem 3.3 Assume that $M_{1}^{2} L<1$ holds, and $:[0,+\infty) \times \mathbf{R} \rightarrow \mathbf{R}$ be an $L^{2}$-Carathéodory function such that $\left(\mathrm{S}^{\prime}\right)$ satisfies, and $f(t, u) \geq 0$ for all $(t, u) \in[0,+\infty) \times \mathbf{R}$. Furthermore, suppose that there exist a function $\omega \in X$ and two positive constants $c_{1}, c_{2}$ with $\frac{4 c_{1}}{1-M_{1}^{2} L}<$ $\|\omega\|_{X}^{2}<\frac{c_{2}}{1+M_{1}^{2} L}$ such that

$$
\frac{\int_{0}^{+\infty} \sup _{|u| \leq \sqrt{\theta c_{1}}} F(t, u) d t}{c_{1}}<\frac{4 \int_{0}^{+\infty} F(t, \omega(t)) d t}{3\left(1+M_{1}^{2} L\right)\|\omega\|_{X}^{2}}
$$

$$
\frac{\int_{0}^{+\infty} \sup _{|u| \leq \sqrt{\theta c_{2}}} F(t, u) d t}{c_{2}}<\frac{2 \int_{0}^{+\infty} F(t, \omega(t)) d t}{3\left(1+M_{1}^{2} L\right)\|\omega\|_{X}^{2}} .
$$

Then, for each $\lambda$ in

$$
\begin{aligned}
\Lambda_{2}:= & \left(\frac{3\left(1+M_{1}^{2} L\right)\|\omega\|_{X}^{2}}{4 \int_{0}^{+\infty} F(t, \omega(t)) d t}, \min \left\{\frac{c_{1}}{\int_{0}^{+\infty} \sup _{|u| \leq \sqrt{\theta c_{1}}} F(t, u) d t},\right.\right. \\
& \left.\left.\frac{c_{2}}{2 \int_{0}^{+\infty} \sup _{|u| \leq \sqrt{\theta c_{2}}} F(t, u) d t}\right\}\right),
\end{aligned}
$$


and for every negative continuous function $I_{j}, j=1,2, \ldots, p$, and $g(u) \leq 0$ for all $u \in \mathbf{R}$, there exists

$$
\delta_{2}:=\min \left\{\frac{\lambda \int_{0}^{+\infty} \sup _{|u| \leq \sqrt{\theta c_{1}}} F(t, u) d t-c_{1}}{I_{\sqrt{\theta c_{1}}}^{*}}, \frac{\lambda \int_{0}^{+\infty} \sup _{|u| \leq \sqrt{\theta c_{2}}} F(t, u) d t-\frac{c_{2}}{2}}{I_{\sqrt{\theta c_{2}}}^{*}}\right\}
$$

such that, for each $\mu \in\left[0, \delta_{2}\right), I B V P(1.1)$ has three distinct classical solutions $u_{i}, i=1,2,3$, with $0<\left\|u_{i}\right\|_{X} \leq \sqrt{\frac{2 c_{2}}{1-M_{1}^{2} L}}$.

Proof In order to apply Theorem 2.4 to IBVP (1.1), we take the functionals $\Phi, \Psi: X \rightarrow \mathbf{R}$ as given in (2.3) and (2.4). Obviously, $\Phi$ and $\Psi$ satisfy the conditions (1) of Theorem 2.4. Now we will prove that the functional $E_{\lambda}(u)=\Phi(u)-\lambda \Psi(u)$ satisfies the assumption (2) of Theorem 2.4. Let $u^{*}$ and $u^{* *}$ be two local minima for $E_{\lambda}(u)$. Then $u^{*}$ and $u^{* *}$ are critical points for $E_{\lambda}(u)$, which implies that $u^{*}, u^{* *}$ are weak solutions of IBVP (1.1). In particular $u^{*}$ and $u^{* *}$ are nonnegative. Indeed, with no loss of generality we may assume that $u_{0}$ be a weak solution of IBVP $(1.1)$, and the set $\Omega=\left\{t \in(0,+\infty): u_{0}(t)<0\right\}$ is nonempty and of positive measure. Furthermore, taking into account that $u_{0}$ is a weak solution, one has

$$
\int_{0}^{+\infty} u_{0}^{\prime \prime}(t) v(t) d t-m^{2} \int_{0}^{+\infty} u_{0}(t) v(t) d t+\lambda \int_{0}^{+\infty} f\left(t, u_{0}(t)\right) v(t) d t=0
$$

for all $v \in X$.

Put $\bar{v}(t)=\max \left\{0,-u_{0}(t)\right\}$ for all $t \in[0,+\infty)$. Clearly $\bar{v} \in X$ and we deduce that

$$
\begin{aligned}
0= & \sum_{j=0}^{p} \int_{t_{j}}^{t_{j+1}} u_{0}^{\prime \prime}(t) \bar{v}(t) d t-m^{2} \int_{0}^{+\infty} u_{0}(t) \bar{v}(t) d t+\lambda \int_{0}^{+\infty} f\left(t, u_{0}(t)\right) \bar{v}(t) d t \\
= & \left.\sum_{j=0}^{p} u_{0}^{\prime}(t) \bar{v}(t)\right|_{t_{j}} ^{t_{j+1}}-\int_{0}^{+\infty} u_{0}^{\prime}(t) \bar{v}^{\prime}(t) d t-m^{2} \int_{0}^{+\infty} u_{0}(t) \bar{v}(t) d t \\
& +\lambda \int_{0}^{+\infty} f\left(t, u_{0}(t)\right) \bar{v}(t) d t \\
= & -\sum_{j=1}^{p} \triangle u_{0}^{\prime}\left(t_{j}\right) \bar{v}\left(t_{j}\right)-\int_{0}^{+\infty} u_{0}^{\prime}(t) \bar{v}^{\prime}(t) d t-m^{2} \int_{0}^{+\infty} u_{0}(t) \bar{v}(t) d t \\
& +\lambda \int_{0}^{+\infty} f\left(t, u_{0}(t)\right) \bar{v}(t) d t-g\left(u_{0}(0)\right) \bar{v}(0) \\
= & -\mu \sum_{j=1}^{p} \triangle I_{j}\left(u_{0}\left(t_{j}\right)\right) \bar{v}\left(t_{j}\right)+\int_{0}^{+\infty}\left(\bar{v}^{\prime}(t)\right)^{2} d t+m^{2} \int_{0}^{+\infty}(\bar{v}(t))^{2} d t \\
& +\lambda \int_{0}^{+\infty} f\left(t, u_{0}(t)\right) \bar{v}(t) d t-g\left(u_{0}(0)\right) \bar{v}(0) \\
\geq & \|\bar{v}\|^{2},
\end{aligned}
$$

which implies $\bar{v}(t)=0$ for $t \in[0,+\infty)$. Hence, $u_{0} \equiv 0$ on $\Omega$, which is absurd. Then we obtain $u^{*}(t) \geq 0, u^{* *}(t) \geq 0$ for all $t \in[0,+\infty)$. So, one has $s u^{*}+(1-s) u^{* *} \geq 0$ for every $s \in[0,1]$, which implies that

$$
f\left(t, s u^{*}(t)+(1-s) u^{* *}(t)\right) \geq 0
$$


and

$$
I_{j}\left(s u^{*}\left(t_{j}\right)+(1-s) u^{* *}\left(t_{j}\right)\right) \leq 0, \quad j=1,2, \ldots, p .
$$

Consequently, $\Psi\left(s u^{*}+(1-s) u^{* *}\right) \geq 0$ for every $s \in[0,1]$.

From (3.4) and (3.5), we have

$$
\frac{1-M_{1}^{2} L}{2}\|u\|_{X}^{2} \leq \Phi(u) \leq \frac{1+M_{1}^{2} L}{2}\|u\|_{X}^{2},
$$

for all $u \in X$.

Note that by the condition $\frac{4 c_{1}}{1-M_{1}^{2} L}<\|\omega\|^{2}<\frac{c_{2}}{1+M_{1}^{2} L}$, we get $2 c_{1}<\Phi(\omega)<\frac{c_{2}}{2}$. It follows from the definition of $\Phi,(2.1)$, and (3.10) that

$$
\begin{aligned}
\Phi^{-1}(-\infty, r) & =\{u \in X: \Phi(u)<r\} \\
& \subseteq\left\{u \in X:\|u\|_{X}<\sqrt{\frac{2 r}{1-M_{1}^{2} L}}\right\} \\
& \subseteq\left\{u \in X:\|u\|_{\infty}<\sqrt{\frac{2 M_{1}^{2} r}{1-M_{1}^{2} L}}\right\} \\
& =\{u \in X:|u(t)|<\sqrt{\theta r} \text { for all } t \in[0,+\infty)\} .
\end{aligned}
$$

So, we have

$$
\begin{aligned}
\sup _{u \in \Phi^{-1}(-\infty, r)} \Psi(u) & =\sup _{u \in \Phi^{-1}(-\infty, r)}\left\{\int_{0}^{+\infty} F(t, u(t)) d t-\frac{\mu}{\lambda} \sum_{j=1}^{p} \int_{0}^{u\left(t_{j}\right)} I_{j}(s) d s\right\} \\
& \leq \int_{0}^{+\infty} \sup _{|u| \leq \sqrt{\theta r}} F(t, u(t)) d t-\frac{\mu}{\lambda} I_{\sqrt{\theta r}}^{*} .
\end{aligned}
$$

Therefore, due to the assumption (D1), one gets

$$
\begin{aligned}
\frac{1}{c_{1}} \cdot \sup _{u \in \Phi^{-1}\left(-\infty, c_{1}\right)} \Psi(u) & \leq \frac{\int_{0}^{+\infty} \sup _{|u| \leq \sqrt{\theta c_{1}}} F(t, u(t)) d t-\frac{\mu}{\lambda} I_{\sqrt{\theta c_{1}}}^{*}}{c_{1}} \\
& <\frac{1}{\lambda} \leq \frac{4}{3\left(1+M_{1}^{2} L\right)} \cdot \frac{\int_{0}^{+\infty} F(t, \omega(t)) d t}{\|\omega\|_{X}^{2}} \\
& \leq \frac{2}{3} \frac{\Psi(\omega)}{\Phi(\omega)} .
\end{aligned}
$$

From the assumption (D2), one infers that

$$
\begin{aligned}
\frac{1}{c_{2}} \cdot \sup _{u \in \Phi^{-1}\left(-\infty, c_{2}\right)} \Psi(u) & \leq \frac{\int_{0}^{+\infty} \sup _{|u| \leq \sqrt{\theta c_{2}}} F(t, u(t)) d t-\frac{\mu}{\lambda} I_{\sqrt{\theta c_{2}}}^{*}}{c_{2}} \\
& <\frac{1}{2 \lambda} \leq \frac{2}{3\left(1+M_{1}^{2} L\right)} \cdot \frac{\int_{0}^{+\infty} F(t, \omega(t)) d t}{\|\omega\|_{X}^{2}} \\
& \leq \frac{1}{3} \frac{\Psi(\omega)}{\Phi(\omega)} .
\end{aligned}
$$


So, the conditions (b1) and (b2) of Theorem 2.4 are satisfied. Then by means of Theorem 2.4, IBVP (1.1) admits at least three distinct weak solutions $u_{i}(i=1,2,3)$ in $X$, such that $0<\left\|u_{i}\right\|_{X} \leq \sqrt{\frac{2 c_{2}}{1-M_{1}^{2} L}}$. This completes the proof.

Example 3.4 Consider the following boundary value problem with impulsive effect:

$$
\left\{\begin{array}{l}
-u^{\prime \prime}(t)+\frac{1}{2} u(t)=\lambda f(t, u(t)), \quad t \in[0,+\infty), t \neq t_{1}, \\
\triangle\left(u^{\prime}\left(t_{1}\right)\right)=\mu I_{1}\left(u\left(t_{1}\right)\right), \\
u^{\prime}\left(0^{+}\right)=g(u(0)), \quad u^{\prime}(+\infty)=0
\end{array}\right.
$$

where $m=\frac{\sqrt{2}}{2}, I_{1}(u)=2-\sqrt{\left|u\left(t_{1}\right)\right|}$. Choose $\alpha_{1}=2, \beta_{1}=1, \sigma_{1}=\frac{1}{2}$, then the condition $(\mathrm{C} 0)$ is satisfied. $M_{1}$ is a positive constant defined in (2.1). When $M_{1}$ lies in different intervals, we can choose different $f$ and $g$ satisfying the conditions. Hence we only consider one case. If $M_{1} \leq \frac{\sqrt{2}}{4}$, we may choose $g(x)=\frac{1}{4 M_{1}^{2}} \sin x$ and

$$
f(t, u)= \begin{cases}M_{1} e^{-t}, & u \leq \frac{M_{1}}{2}, \\ \frac{4 e^{-t} u^{2}}{M_{1}}, & \frac{M_{1}}{2}<u \leq M_{1} \\ \frac{4 e^{-t} M_{1}^{2}}{u}, & u>M_{1}\end{cases}
$$

where $M_{1}$ is given in (2.1). Then

$$
F(t, u)= \begin{cases}M_{1} e^{-t} u, & u \leq \frac{M_{1}}{2}, \\ \frac{4 e^{-t} u^{3}}{3 M_{1}}+\frac{M_{1}^{2} e^{-t}}{3}, & \frac{M_{1}}{2}<u \leq M_{1}, \\ 4 e^{-t} M_{1}^{2} \ln u+\left(\frac{5}{3}-\ln M_{1}\right) M_{1}^{2} e^{-t}, & u>M_{1} .\end{cases}
$$

Obviously, $f$ satisfies $\left(\mathrm{S}^{\prime}\right)$ with $l(t)=4 M_{1} e^{-t}$, and $g$ satisfies (1.2) with $L=\frac{1}{4 M_{1}^{2}}$, and $M_{1}^{2} L<1$. Take $a=\frac{M_{1}^{2}}{2}, b=M_{1}$. By simple calculations (C1) and (C2) are satisfied. Applying Theorem 3.2, IBVP (3.11) admits at least three distinct classical solutions for each $\lambda \in\left(\frac{24}{35}, \frac{3 \sqrt{2}}{16 M_{1}}\right)$, and for each

$$
0<\mu<\min \left\{\frac{9-24 \sqrt{2} \lambda M_{1}}{\left(48+8 \sqrt{2} M_{1}\right) M_{1}^{2}}, \frac{(35 \lambda-24) M_{1}}{96+32 \sqrt{M_{1}}}\right\} .
$$

Example 3.5 Consider the following problem:

$$
\left\{\begin{array}{l}
-u^{\prime \prime}(t)+u(t)=\lambda f(t, u(t)), \quad t \in[0,+\infty), t \neq t_{j}, \\
\triangle\left(u^{\prime}\left(t_{1}\right)\right)=\mu I_{1}\left(u\left(t_{1}\right)\right), \\
u^{\prime}\left(0^{+}\right)=g(u(0)), \quad u^{\prime}(+\infty)=0
\end{array}\right.
$$

where $m=1, I_{1}(u)=-1-\left|u\left(t_{1}\right)\right|$. Then $I_{1}(u) \leq 0$ for any $u \in \mathbf{R}$. $M_{1}$ is a positive constant defined in (2.1). When $M_{1}$ lies in different intervals, we can choose different $f$ and $g$ satisfying the conditions. For example, if $\frac{\sqrt{3}}{48} \leq M_{1}<1$, we may choose $g(x)=-\frac{1-M_{1}^{2}}{M_{1}^{2}}(1-\cos x)$ and

$$
f(t, u)= \begin{cases}u^{2} e^{-t}, & u \leq 4 M_{1} \\ \frac{256 M_{1}^{4} e^{-t}}{u^{2}}, & u>4 M_{1}\end{cases}
$$


where $M_{1}$ is given in (2.1). Then

$$
F(t, u)= \begin{cases}\frac{1}{3} u^{3} e^{-t}, & u \leq 4 M_{1}, \\ -\frac{256 M_{1}^{4} e^{-t}}{u}+\frac{256 M_{1}^{3} e^{-t}}{3}, & u>4 M_{1} .\end{cases}
$$

Obviously, $M_{1}^{2} L<1, f(t, u) \geq 0$ for all $(t, u) \in[0,+\infty) \times \mathbf{R}$ and satisfies (S3') with $l(t)=$ $16 M_{1}^{2} e^{-t} . g$ satisfies (1.2) with $L=\frac{1-M_{1}^{2}}{M_{1}^{2}}>0$, and $g(u) \leq 0$ for any $u \in \mathbf{R}$. Take $\omega(t)=4 M_{1} e^{-t}$, $c_{1}=\frac{M_{1}^{2}}{288}$, and $c_{2}=800 M_{1}^{2}$. Then $\theta=2, \frac{4 c_{1}}{1-M_{1}^{2} L}<\|\omega\|_{X}^{2}=16 M_{1}^{2}<\frac{c_{2}}{1+M_{1}^{2} L}$. By simple calculations (D1) and (D2) are satisfied. Applying Theorem 3.2, for each $\lambda \in\left(\frac{18-9 M_{1}^{2}}{4 M_{1}}, \frac{18}{M_{1}}\right)$ and for each

$$
0<\mu<\min \left\{\frac{M_{1}\left(1-\frac{\lambda M_{1}}{18}\right)}{M_{1}+24}, \frac{10 M_{1}\left(1-\frac{18 \lambda M_{1}}{125}\right)}{20 M_{1}+1}\right\}
$$

IBVP (3.12) admits at least three distinct classical solutions $u_{i}(i=1,2,3)$ with $0<\left\|u_{i}\right\|_{X} \leq$ 40 .

We observe that in Example 3.4 and Example 3.5 the functions $f$, $g$, and the impulsive term do not satisfy the conditions $(\mathrm{H} 1),(\mathrm{H} 2)$ of Theorem 3.1 in [17] or the conditions of Theorem 3.2 in [25]. Hence, the problem (3.11) and (3.12) cannot be dealt with by the results of $[17,25]$.

\section{Competing interests}

The authors declare that they have no competing interests.

\section{Authors' contributions}

The authors declare that the study was realized in collaboration with the same responsibility. All authors read and approved the final manuscript.

\section{Acknowledgements}

The authors are highly grateful for the referees' careful reading and comments on this paper. The research is supported by Hunan Provincial Natural Science Foundation of China (No. 13JJ3106).

Received: 18 September 2014 Accepted: 12 November 2014 Published online: 28 November 2014

\section{References}

1. Agarwal, RP, O’Regan, D: Infinite Interval Problems for Differential, Difference and Integral Equations. Kluwer Academic, Dordrecht (2001)

2. Struwe, M: Variational Methods: Applications to Nonlinear Partial Differential Equations and Hamiltonian Systems. Springer, Berlin (2008)

3. Eloe, PW, Kaufmann, ER, Tisdell, CC: Multiple solutions of a boundary value problem on an unbounded domain. Dyn. Syst. Appl. 15(1), 53-63 (2006)

4. Lian, H, Wang, P, Ge, W: Unbounded upper and lower solutions method for Sturm-Liouville boundary value problem on infinite intervals. Nonlinear Anal. TMA 70, 2627-2633 (2009)

5. Yan, B, O'Regan, D, Agarwal, RP: Unbounded solutions for singular boundary value problems on the semi-infinite interval: upper and lower solutions and multiplicity. J. Comput. Appl. Math. 197, 365-386 (2006)

6. Zhao, Y, Chen, $\mathrm{H}, \mathrm{Xu}, \mathrm{C}$ : Existence of multiple solutions for three-point boundary-value problems on infinite intervals in Banach spaces. Electron. J. Differ. Equ. 2012, 44 (2012)

7. Chen, S, Zhang, Y: Singular boundary value problems on a half-line. J. Math. Anal. Appl. 195, 449-468 (1995)

8. Liu, B, Li, J, Liu, L: Existence and uniqueness for an $m$-point boundary value problem at resonance on infinite intervals. Comput. Math. Appl. 64, 1677-1690 (2012)

9. Lakshmikantham, V, Bainov, DD, Simeonov, PS: Theory of Impulsive Differential Equations. World Scientific, Singapore (1989)

10. Benchohra, M, Henderson, J, Ntouyas, S: Theory of Impulsive Differential Equations. Contemporary Mathematics and Its Applications. Hindawi Publishing Corporation, New York (2006)

11. Liu, L, Liu, Z Wu, Y: Infinite boundary value problems for $n$ th-order nonlinear impulsive integro-differential equations in Banach spaces. Nonlinear Anal. TMA 67, 2670-2679 (2007)

12. Yan, B: Boundary value problems on the half-line with impulsive and infinite delay. J. Math. Anal. Appl. 259, 94-114 (2001) 
13. Kaufmann, ER, Kosmatov, N, Raffoul, YN: A second-order boundary value problem with impulsive effects on an unbounded domain. Nonlinear Anal. TMA 69, 2924-2929 (2008)

14. Li, J, Nieto, JJ: Existence of positive solutions for multiple boundary value problems on the half-line with impulsive. Bound. Value Probl. 2009, Article ID 834158 (2009)

15. Gomes, M, Sanchez, L: A variational approach to some boundary value problems in the half-line. Z. Angew. Math. Phys. 56, 192-209 (2005)

16. Nieto, JJ, O'Regan, D: Variational approach to impulsive differential equations. Nonlinear Anal., Real World Appl. 10, 680-690 (2009)

17. Chen, $\mathrm{H}$, Sun, J: An application of variational method to second-order impulsive differential equation on the half-line. Appl. Math. Comput. 217, 1863-1869 (2010)

18. Bonanno, G, Marano, SA: On the structure of the critical set of non-differentiable functions with a weak compactness condition. Appl. Anal. 89, 1-10 (2010)

19. Sun, J, Chen, H, Nieto, JJ, Otero-Novoa, M: Multiplicity of solutions for perturbed second-order Hamiltonian systems with impulsive effects. Nonlinear Anal. TMA 72, 4575-4586 (2010)

20. Sun, J, Chen, H: Multiple positive solutions for multi-point boundary value problems with a $p$-Laplacian on the half-line. J. Appl. Math. Comput. 33, 173-191 (2010)

21. Zhang, H, Li, Z: Periodic and homoclinic solutions generated by impulses. Nonlinear Anal., Real World Appl. 12, 39-51 (2011)

22. Chen, $P$, Tang, X: Existence and multiplicity of solutions for second-order impulsive differential equations with Dirichlet problems. Appl. Math. Comput. 218, 11775-11789 (2012)

23. Tian, Y, Ge, W: Variational methods to Sturm-Liouville boundary value problem for impulsive differential equations. Nonlinear Anal. TMA 72, 277-287 (2010)

24. Tian, Y, Ge, W: Multiple solutions of impulsive Sturm-Liouville boundary value problem via lower and upper solutions and variational methods. J. Math. Anal. Appl. 387, 475-489 (2012)

25. Dai, B, Zhang, D: The existence and multiplicity of solutions for second-order impulsive differential equations on the half-line. Results Math. 63, 135-149 (2013)

26. Ricceri, B: A general variational principle and some of its applications. J. Comput. Appl. Math. 113, 401-410 (2000)

27. Xiao, J, Nieto, JJ, Luo, Z: Multiplicity of solutions for nonlinear second order impulsive differential equations with linear derivative dependence via variational methods. Commun. Nonlinear Sci. Numer. Simul. 17, 426-432 (2012)

28. $\mathrm{Xu}, \mathrm{J}, \mathrm{Wei}, \mathrm{Z}$, Ding, Y: Existence of weak solutions for $p$-Laplacian problem with impulsive effects. Taiwan. J. Math. 17(2), 501-515 (2013)

29. Mawhin, J, Willem, M: Critical Point Theory and Hamiltonian Systems. Springer, Berlin (1989)

30. Bonanno, G, Riccobono, G: Multiplicity results for Sturm-Liouville boundary value problems. Appl. Math. Comput. 210, 294-297 (2009)

31. Zou, W: Variant fountain theorems and their applications. Manuscr. Math. 104, 343-358 (2001)

32. Brézis, H: Analyse Fonctionelle-Théorie et Applications. Masson, Paris (1983)

33. Zeidler, E: Nonlinear Functional Analysis and Its Applications. III. Springer, Berlin (1985)

34. Bonanno, G, Candito, P: Non-differentiable functionals and applications to elliptic problems with discontinuous nonlinearities. J. Differ. Equ. 244, 3031-3059 (2008)

35. Zeidler, E: Nonlinear Functional Analysis and Its Applications. II/B. Springer, Berlin (1990)

doi:10.1186/s13661-014-0246-8

Cite this article as: Zhao et al.: New results for perturbed second-order impulsive differential equation on the half-line. Boundary Value Problems 2014 2014:246.

\section{Submit your manuscript to a SpringerOpen ${ }^{\circ}$ journal and benefit from:}

- Convenient online submission

- Rigorous peer review

- Immediate publication on acceptance

- Open access: articles freely available online

- High visibility within the field

- Retaining the copyright to your article 